vision in the right eye to only hand movement at 3 feet, but the corrected vision in the left eye was $20 / 30$. There was no anterior segment neovascularisation and the anterior chamber angle was grade IV open. The intraocular pressures were normal in both eyes. Funduscopic examination revealed typical finding of ischaemic CRVO, which was supported by fluorescein angiography as shown in Figure 1. Laboratory investigations at the time of the diagnosis showed haemoglobin of $7.9 \mathrm{~g} / \mathrm{l}$, haematocrit of $26.4 \%$, WBC of $800 \times 10^{\circ} / 1$, and platelets of $30 \times 10^{\%} / 1$. Extensive coagulation investigation including antithrombin III, heparin-cofactor II and protein S, protein $\mathrm{C}$, antiphospholipid antibodies, and circulating immune complexes (CIC), did not show any abnormalities.

The patient was followed without treatment for 1 year, her vision remained unchanged in the left eye, but showed some improvement in the right eye; she was able to count fingers at 1 foot.

\section{Comment}

This is the second case in the literature of CRVO causing visual loss in a patient with AIDS. CMV retinitis remains the most common cause of loss of vision in AIDS patients. ' The prognosis and the treatment of CRVO and CMV retinitis are quite different. ${ }^{13}$ The diagnosis in our patient was suspected on funduscopic examination and supported by fluorescein angiography (Fig 1). CRVO occurred in this patient in the absence of underlying cardiovascular disease or any significant coagulation defects that can lead to thrombosis. ${ }^{2+}$ As in the previously reported case, our patient developed CRVO in conjunction with the treatment of the anaemia. In the present case, the anaemia was treated with r-HuEPO. Thrombosis of arteriovenous fistulas and native veins has been reported in patients in chronic haemodialysis during the course of treatment with r-HuEPO.' The haematocrit did not rise significantly in this case $(24 \%$ to $26.4 \%)$, however, other rheological effect of r-HuEPO such as increased platelet aggregation could still contribute to CRVO in AIDS patients who are likely to have an abnormal retinal microcirculation..$^{\text {is }}$

Finally, CVRO in this case, as well as in the case presented by Tiech et $a l,{ }^{2}$ occurred in patients known to have AIDS. It remains unclear as to whether or not the CRVO may precede significant AIDS related illness.

We thank Jan Isaacs for her secretarial skills.

1 Palestine AG, Polis MA, Daomet MD, Baird BF, Falloon J, Kovacs JA, et al. A randomized, controlled trial of foscarne in the treatment of cytomegalovirus retinitis in patients with AIDS. Ann Intern Med 1991; 115: 665-73.

2 Tiech SA, Sonnabend J. Central retinal vein occlusion in a patient with AIDS. Arch Ophthalmol 1988; 106: 1508-9.

3 Kohner EM, Laatikaineu L, Oughton J. The management of central retinal vein occlusion (CRVO). Ophthalmology 1983; central retinal 484 .

4 Cansos RT, Zon LI, Groopman JE. Anticardiolipin antibodies associated with HTLV-III infection. BrF Haematol 1987; 65: associa 8 .

5 Castasi S, Passerini P, Campise MR, Graziani G, Cesana B, Perisic $M$, et al. Benefits and risks of protracted treatment with human recombinant erythropoietin in patients having hemodialysis. $B M \mathcal{F}$ 1987; 295: 1017-20.

\title{
Persistent visual changes following hashish consumption
}

\author{
Gian Luca Laffi, Avinoam B Safran
}

Neuro-ophthalmology Unit, Department of Ophthalmology, Geneva University Hospital, Geneva, Switzerland G L Laffi A B Safran

${ }^{\star}$ Current address: Department of Ophthalmology, Ottica Fisiopatologica (Pf Meduri) Bologna University Hospital Bologna, Italy.

Correspondence to: Professor Avinoam B Safran, Neuro-ophthalmolgy Unit, Geneva University Hospital 1211 Geneva 4, Switzerland.

Accepted for publication 28 April 1993
Lasting changes in vision following drug misuse were recently described by Levi and Miller. ${ }^{.}$In one of the reported patients, visual alterations occurred as a result of marijuana misuse only. We observed a subject who presented visual changes which started the day following discontinuation of a 5 year long period of continuous hashish consumption, and which had lasted for months. Visual symptoms were strikingly increased on physical effort or when reading from a brightly lit paper.

Our observation corroborates the recent suggestion by Levi and Miller,' that lasting, harmful effects of cannabinoid consumption on visual function may have been previously underestimated.

\section{Case report}

A 23-year-old man complained of visual changes lasting for 8 months. Although mentally rather well balanced, when aged 18 he started smoking hashish for social reasons, and went on smoking several times a day, uninterrupted for 5 years. He denied having used any other drug. On the day following discontinuation of drug misuse, he noted the occurrence of changes in visual function, consisting mainly in perception of small black and white spots flickering randomly at high frequency. The phenomenon was described as similar to interference on a television screen. These visual phenomena were markedly increased by either starting physical effort or reading from a brightly lit white paper, making everyday life difficult. The patient also described a reduction in capacity for mental concentration, and in depth perception, and the occurrence of visual perseveration after looking at bright objects. In addition, he occasionally noted a feeling of disconnection between himself and 
people with whom he was speaking. Headaches and changes in concentration abilities resolved within 4 months, whereas visual symptoms still persisted after 8 months of drug abstinence, although slightly reduced in intensity.

Neuro-ophthalmic evaluation, including visual acuity, visual fields, stereopsis using the Titmus test, colour perception, and pattern visually evoked response, failed to demonstrate any abnormality. Neurological examination was normal, including electroencephalogram and computed tomography scan.

\section{Comment}

Disturbances of visual perception following cannabinoid consumption are poorly defined. In the report by Levi and Miller,' a patient who misused marijuana alone, like ours, experienced visual changes, including alteration in depth perception and some kind of sensorial disconnection when talking to people. In addition, both subjects experienced intermittent light phenomena; in one as a strobe-like effect, and in the other as bright spots flickering randomly at high frequency.

In our patient, increasing the brightness of the background, even if this was only reading from a bright white paper, enhanced the visual symptoms. In a number of conditions, abnormal sensory phenomena increase as background luminance increases - for example, pain during attacks of migraine, or dazzling in subjects with optic neuropathy. Various mechanisms might be considered as explaining the influence of ambient light, including opticotrigeminal summation ${ }^{2}$ in migraine headache, and intermittent blocks in nerve conduction ${ }^{3}$ in optic neuropathy. In our observation, mechanisms by which visual alterations are increased with a light background are still poorly understood. As concentration was altered, it is conceivable that cortical disinhibition was involved.

Visual abnormalities were also increased by stress in the patient described by Levi and Miller, and by physical effort in ours. It is possible that changes in vasomotor control, which have been shown to occur following cannabinoid misuse, ${ }^{4}$ might play a role in such circumstances.

The visual changes in our patient appeared on the day following discontinuation of hashish misuse. This indicates that, following cannabinoid consumption as with other drugs, ${ }^{1}$ persistent changes in vision can occur after a drug-free interval. Lasting effects of cannabinoid misuse are more frequent than would appear from published reports. However, this diagnosis should only be made after other causes of nonspecific alteration in visual perception have been excluded by a careful history and by medical and neuroimaging evaluation.

\footnotetext{
1 Levi L, Miller NR. Visual illusions associated with previous drug abuse. 7 Clin Neuro-ophthalmol 1990; 10: 103-10.

2 Eckardt LB, McLean JM, Goodell H. Experimental studies on headache: the genesis of pain from the eye. In: Proceedings of the Association for Research in Nervous and Memtal Diseases. Baltimore: Williams \& Wilkins, 1943; 23: 209-27.

3 Safran AB, Bader C, Brazitikos P, de Weisse C, Désangles D. Increasing short-term fluctuation by increasing the intensity of the fixation aid during perimetry. Am f Ophthalmol 1992; 113: 193-7.

4 Katzung BG, Trevor AJ. Pharmacology. 2nd ed. San Francisco: Appleton and Lange, 1989: 167-73.
}

\title{
Dirofilariasis: an uncommon parasitosis of the eye
}

\author{
Merih Soylu, Kadri Özcan, Müslime Yalaz, Seyhan Varinli, Gülhan Slem
}

University of Çukurova

Medical Faculty, Adana,

Turkey

Department of

Ophthalmology

M Soylu

\section{Department of Parasitology K Özcan}

Department of Ophthalmology M Yalaz

Department of Pathology $S$ Varinli

G Slem

Correspondence to:

Dr M Soylu, Cukurova

University Medical Faculty,

Department of

Ophthalmology, Balcal,

Adana, Turkey.

Accepted for publication

19 March 1993
Dirofilariasis, a disease rarely seen in humans, occurs commonly in carnivorous mammals. Two important species in the Dirofilaria genus are found in the eye. One of these is $D$ tenuis, whose natural host is raccoons. As there are no raccoons in Turkey, this parasite is not found there. The other is $D$ repens, which is found in dogs and cats. Since only immature forms are found in humans, no microfilarias are detected in the circulation. ${ }^{12}$ Although it is an uncommon parasite in humans, when found it is usually located in the subepithelial tissues of the eyelids, fingers, cheeks, breasts, abdomen, and very rarely in conjunctivae. The source and the route of infection is not known definitely. Diagnosis could only be made by detecting the parasite in the histological specimen. Treatment is only by surgical excision. ${ }^{1-4}$

\section{Case reports}

CASE 1

A 22-year-old man, complaining of photophobia, redness, and conjunctival swelling in the left eye of 1 week's duration, was admitted to hospital. In the ophthalmic evaluation a ciliary injection and a superotemporally located subconjunctival mass were detected (Fig 1). The mass contained a thin, white living worm. Routine blood tests were within normal limits, and no microfilaria were detected in the blood smear. The worm was removed with a forceps, the subconjunctival mass was excised with the conjunctiva, and the exposed sclera was covered by Tenon's capsule under local anaesthesia. The specimen measured $1 \times 0.5 \mathrm{~cm}$ and histopathologically exhibited a mixed inflammatory 